BOSQUE 26(2): 47-56, 2005

NOTA TECNICA

\title{
Ensayo de una tipología de estaciones forestales en el Parque Nacional Tolhuaca, Chile
}

\author{
An essay on a forest stations typology in the Tolhuaca National Park, Chile \\ RODOLFO GAJARDO, FEDERICO LUEBERT, MARLIS ESTAY \\ Universidad de Chile, Facultad de Ciencias Forestales, Departamento de Silvicultura, \\ Casilla 9206, Santiago, Chile
}

\begin{abstract}
SUMMARY
A typology of forest stations is established for the Tolhuaca National Park, Chile. A forest station is a homogenous environmental unit, defined through its physical and biotical characteristics. In order to establish the typology of stations, three methodological stages were achieved, which are the definition of phytosociological and phytoecological groups and types of physical environments or biotopes. The identified phytosociological associations are distributed in the classes Wintero-Nothofagetea, with three associations, and Nothofagetea pumilionisantarcticae, also with three associations. The results obtained with the use of the factorial analysis of correspondences, allow establishing four phytoecological groups: mesophyllous deciduous forest, with broad-leaved evergreen species; microphyllous deciduous forest; microphyllous evergreen forest and mixed microphyllous deciduous scrub, with evergreen species. The best variables for the definition of the biotopes are altitude and relief, both factors with great influence on the local climate. The studied area is located within the temperate macroclimate and the following belts were defined: colinen, lower montane and upper montane. The proposed typology of forest stations consists of fourteen units.
\end{abstract}

Key words: forest stations, forest typology, Tolhuaca National Park.

\section{RESUMEN}

Se establece una tipología de estaciones forestales para el Parque Nacional Tolhuaca, Chile. El concepto de estación forestal se refiere a una unidad ambiental homogénea, definida a través del conjunto de sus caracteres físicos y bióticos. En orden a establecer la tipología de estaciones se cumplieron tres etapas metodológicas, las cuales son: la definición de grupos fitosociológicos, grupos fitoecológicos y tipos de ambiente físico o biótopos. Los grupos fitosociológicos determinados están distribuidos en la clase Wintero-Nothofagetea, con tres asociaciones, y en la clase Nothofagetea pumilionis-antarcticae, también con tres asociaciones. Los resultados obtenidos con el uso del análisis factorial de correspondencias permiten establecer cuatro grupos fitoecológicos, correspondientes a bosque caducifolio mesófilo, con especies: laurifolias, bosque caducifolio micrófilo, bosque siempreverde micrófilo y matorral caducifolio micrófilo, con especies siempreverdes. Las mejores variables determinadas para la definición de los biótopos son altitud y relieve, ambos factores con gran influencia en el clima local. El área estudiada se ubica dentro del macroclima templado y en ella fueron definidos los siguientes pisos: colino, montano inferior y montano superior. La tipología de estaciones forestales propuesta consiste en catorce unidades.

Palabras clave: estaciones forestales, tipología forestal, Parque Nacional Tolhuaca. 
BOSQUE 26(2): 47-56, 2005

Ensayo de una tipología de estaciones forestales en el Parque Nacional Tolhuaca, Chile

\section{INTRODUCCION}

La definición y clasificación de las comunidades vegetales, sobre la base de criterios florísticos, fisionómico-estructurales, biogeográficos y ecológicos, es un tema sobre el cual existen amplias referencias $(1,2,3)$. Pero la práctica de la silvicultura y el manejo de bosques ha buscado la definición de unidades que expresen la productividad potencial del sitio $(4,5,6,7)$. Hills (8) afirma que "las combinaciones del macroclima y el relieve no sólo constituyen la base de la productividad potencial, sino que, además, ejercen un control funcional sobre el desarrollo integrado de la vegetación, del suelo y del microclima". Cajander (9) fue el primero en reconocer la expresión de las condiciones del sitio en la vegetación estable, especialmente a través del análisis de la composición florística del sotobosque. Duchaufour et al. (10) orienta la definición de estación forestal a las correlaciones que existen entre los factores del medio (roca madre, suelo, topografía, clima local) y los factores biológicos (comunidad florística, vegetación). Estas correlaciones permiten definir tipos de bosque, correspondientes a estaciones o sitios, donde cada uno de ellos está caracterizado por un tipo de humus, condicionando un cierto tipo de suelo. Bonneau y Timbal (11) utilizan el concepto de estación forestal para referirse a una unidad biológica intuitiva, correspondiente a un paisaje vegetal homogéneo en cuanto a su composición, situado en una posición topográfica determinada, en el cual se puede practicar una misma silvicultura y es posible esperar una producción similar. Brêthes (12) da mayor precisión a la definición de estación, refiriéndose a ella como una unidad concreta de terreno correspondiente a condiciones ecológicas locales homogéneas, dependientes de la naturaleza de las formaciones geológicas superficiales, del clima y de la topografía; esas condiciones ecológicas particulares se expresan a través del suelo y de la vegetación. El tipo de estación no existe en la realidad del terreno, sino que corresponde a un concepto abstracto que agrupa al conjunto de situaciones análogas por su posición topográfica y geomorfología, la naturaleza del suelo, la composición florística y la dinámica de la vegetación.

En Chile, Donoso $(13,14)$ propone una clasificación de tipos forestales en función de las especies presentes y dominantes que conforman la estructura y los estratos superiores del bosque, considerando la ubicación geográfica de la comunidad forestal. Vita (15) define al rodal como una unidad de gestión en silvicultura, la cual se caracteriza por la uniformidad en la asociación vegetal su estructura y etapa de desarrollo; indica que un rodal no debiera abarcar más que un tipo de estación, refiriéndose en este caso al sitio como biótopo. Perthuisot (16) realiza una tipología de estaciones en la Reserva Nacional Malleco, usando el concepto en el sentido que le da Brêthes (12), estableciendo doce estaciones como base descriptiva de los recursos boscosos disponibles.

La necesidad, cada vez mayor, de una silvicultura intensiva requiere una mejor comprensión de las potencialidades y restricciones que presenta localmente el complejo ambiental y las biocenosis asociadas. Sobre esta base, y frente al escaso desarrollo de estudios de este carácter en Chile, se ha ensayado la metodología propuesta por Brêthes (12), que es de amplia aplicación en la práctica forestal francesa, en la definición de una tipología de estaciones forestales en el Parque Nacional Tolhuaca (17).

\section{MATERIAL Y METODO}

Area de estudio. El Parque Nacional Tolhuaca, con una superficie de 6.374 ha, se encuentra en la precordillera andina de la provincia de Malleco, región de la Araucanía (38 $12^{\prime}$ lat. S, 7141' long. O). En cuanto a la geomorfología, en dirección este-oeste se observa una zona de cordones montañosos, cuyas elevaciones varían entre 1.500 y $1.600 \mathrm{~m} \mathrm{snm}$, entre los cuales existen valles donde se ubican los ríos Malleco y Pichimalleco, con cuencas de origen glacial. Según la clasificación de Köeppen, el clima es templado lluvioso, con cierta sequía relativa de verano (menos de cuatro meses secos) (18); de acuerdo a Amigo y Ramírez (19), el área en estudio se encuentra en el bioclima templado. Según la estación meteorológica más cercana (Los Guindos, $38^{\circ} 03^{\prime}$ lat. S., 71 ${ }^{\circ} 49^{\prime}$ long. O., $440 \mathrm{~m} \mathrm{snm}$ ), la precipitación promedio anual es de $2.482 \mathrm{~mm}$, con una temperatura media anual de $12^{\circ} \mathrm{C}$, con 1 a 2 meses con precipitaciones inferiores a $30 \mathrm{~mm}$. Los suelos son considerados incipientes, sin claros horizontes genéticos, salvo la presencia de horizontes A1 (de acumulación de materia orgánica) y en áreas 
de bosques no intervenidos, aparecen horizontes 01 y 02 (entre 1 y $2 \mathrm{~cm}$ de espesor); son suelos muy estratificados, sin desarrollo de fuertes estructuras (20).

Método de muestreo. Se aplicó un muestreo sistemático, realizado en los meses de marzo y abril de 1999. La malla de muestreo se construyó con un distanciamiento entre transectos de $400 \mathrm{~m}$, ubicados en dirección norte-sur. Las parcelas se realizaron cada $200 \mathrm{~m}$, con una superficie de 200 $\mathrm{m}^{2}$. Las variables registradas se clasificaron en variables físicas: exposición, altitud, pendiente, relieve, erosión, uso actual del suelo y suelo (pedregosidad superficial, textura superficial, profundidad y compactación), y variables florístico-vegetacionales, considerando las estratas arbórea, arbustiva y herbácea, donde se indicaron las especies presentes, evaluada cada una de acuerdo a un índice de abundancia/dominancia y a su forma de vida (3).

Grupos fitosociológicos. La determinación de los grupos fitosociológicos o asociaciones vegetales se realizó aplicando el método de comparación tabular, que agrupa las parcelas considerando el criterio de homogeneidad en la composición, fidelidad y abundancia-dominancia de las especies $(21,22)$. Los resultados del análisis de grupos fitosociológicos son presentados en detalle por Luebert et al. (23).

Grupos fitoecológicos. Se agruparon las asociaciones vegetales en unidades fisionómico-estructurales, combinando variables florísticas y ecológicas. Para ello se utilizaron los resultados entregados por el análisis factorial de correspondencias (AFC), el cual permite interpretar los ejes del plano factorial y determinar así el gradiente ambiental más importante en la distribución de asociaciones vegetales. Los grupos fitoecológicos fueron denominados a partir de la nomenclatura propuesta por Gajardo (24).

Unidades físicas homogéneas o biotopos. Se analizó la matriz inicial de datos (parcelas vs. asociaciones y variables físicas) respecto a los principales factores ambientales que determinan la distribución de las asociaciones obtenidas del AFC. A base de estos factores, se identificaron los grupos de parcelas que comprenden estados simila- res de las variables físicas consideradas para el análisis. En el análisis matricial se consideró la altitud, pues representa una expresión indirecta del clima local. También se registraron la pendiente y la pedregosidad superficial, porque son indicatrices del nivel de humedad, a causa del grado de insolación y del efecto del escurrimiento, y las variables del substrato, ya que indican las posibilidades productivas del medio.

Determinación de estaciones forestales. Las estaciones forestales se determinaron a partir de la relación entre los grupos fitosociológicos y fitoecológicos con respecto a las unidades físicas homogéneas. Para ello se construyó una tabla florística estructurada, cuyas filas corresponden a las unidades ambientales y las columnas a los grupos fitosociológicos y fitoecológicos, ordenados según el factor ambiental principal (12). A base de la tabla y la experiencia de terreno, se determinaron las estaciones forestales presentes, según los procedimientos usados por Pfister y Arno (5) y por Caro (25).

\section{RESULTADOS Y DISCUSION}

Estudio fitosociológico. Se reconocieron seis asociaciones vegetales boscosas (23). La clase Wintero-Nothofagetea $(26,27,28)$ está representada por el orden Laureliopsietalia philippianae, con las alianzas Nothofago-Eucryphion cordifoliae y Nothofago-Winterion; en la primera se ubica la asociación Dasyphyllo diacanthoidis-Nothofagetum alpinae y en la segunda, Nothofagetum procerae. El segundo orden dentro de la clase Wintero-Nothofagetea es Berberido trigonae-Nothofagetalia dombeyi, en el que se ubica la alianza Myrceugenio-Nothofagion dombeyi con la asociación Nothofagetum dombeyi-alpinae. La otra clase presente es Nothofagetea pumilionis-antarcticae $(26,29,30,31)$, con el orden AdenocauloNothofagetalia pumilionis, alianza Nothofagion pumilionis; en ella se ubican las asociaciones Anemono antucensis-Nothofagetum pumilionis y Carici trichodes-Araucarietum araucanae; y el orden Berberido-Nothofagetalia antarcticae, alianza Nothofago antarcticae-Berberidion, con la asociación Lomatio hirsutae-Nothofagetum antarcticae.

La asociación que tiene mayor representación en el territorio del P.N. Tolhuaca corresponde a 
BOSQUE 26(2): 47-56, 2005

Ensayo de una tipología de estaciones forestales en el Parque Nacional Tolhuaca, Chile

Nothofagetum procerae; le siguen Nothofagetum dombeyi-alpinae y Anemono antucensis-Nothofagetum pumilionis.

Estudio fitoecológico. Al agrupar las asociaciones vegetales en unidades fisionómico-estructurales, combinando las variables florísticas con las ecológicas se generaron cuatro grupos fitoecológicos. Esta agrupación tiene como base los resultados del AFC (figura 1). A pesar de que los dos primeros ejes sólo explican el $24 \%$ de la variabilidad del sistema, los resultados son coherentes con lo observado en terreno (17).

La distribución de las asociaciones y variables ambientales en el plano generado por el análisis factorial muestra cuatro grupos. La interpretación del eje primario indica probablemente la expresión de una variable térmica. El polo positivo está ocupado por asociaciones termófilas que crecen en bajas altitudes, como Dasyphyllo diacanthoidis-Nothofagetum alpinae (figura 1, GFE1) y Nothofagetum procerae (figura 1, GFE2); el polo negativo, señalando las altitudes superiores, indica la presencia de Carici trichodes-Araucarietum araucanae y Lomatio hirsutae-Nothofagetum antarcticae (figura 1, GFE4), asociaciones más bien criófilas. El eje secundario, que posiblemente manifiesta la influencia de variables ligadas a una menor disponibilidad hídrica, discrimina en su valor positivo a un grupo constituido por la asociación Anemono antucensis-Nothofagetum pumilionis (figura 1, GFE3) y, en su valor negativo, a otro donde está la asociación Dasyphyllo diacanthoidis-Nothofagetum alpinae (figura 1, GFE1), señalando condiciones de menor a mayor precipitación local (17).

En consecuencia, las unidades fisionómicoestructurales que constituyen los grupos fitoecológicos son las siguientes:

1. Bosque caducifolio mesófilo, con especies caducifolias en el dosel superior, acompañadas por laurifolias en el dosel intermedio y en bosquetes locales. Se encuentra de preferencia en las altitudes menores, en laderas bajas de poca pendiente, en valles húmedos y junto a cursos de agua (figura 1, GFE1).

2. Bosque siempreverde micrófilo, con un dosel superior complejo, denso, donde participan especies micrófilas y laurifolias y caducifolias mesófilas, con matorral secundario en el es-

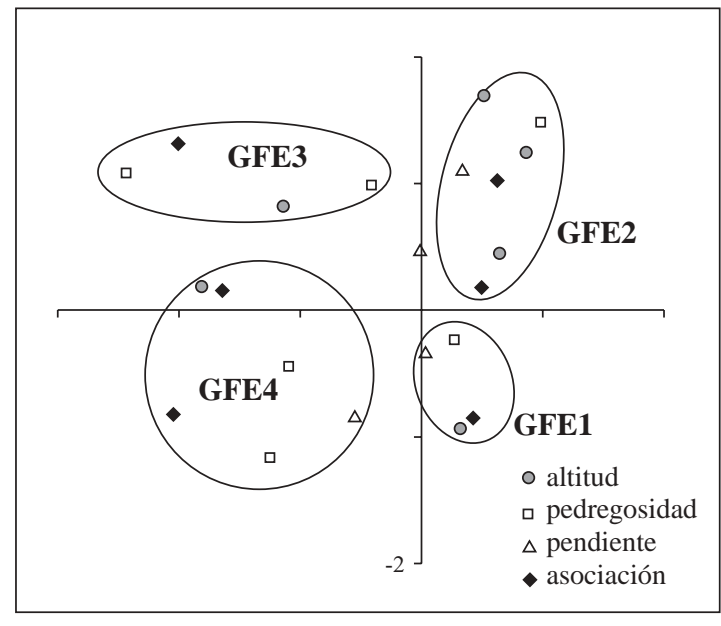

Figura 1. Resultado del AFC, utilizando las variables altitud, pedregosidad, pendiente y asociación vegetal. Los primeros dos ejes factoriales representan el $24 \%$ de la variabilidad del sistema. GFE1: Grupo Fitoecológico 1, asociación Dasyphyllo diacanthoidis-Nothofagetum alpinae, altitud < $700 \mathrm{~m} \mathrm{snm}$, pedregosidad < 1\%, pendiente < 30\%; GFE2: Grupo Fitoecológico 2, asociaciones Nothofagetum procerae y Nothofagetum dombeyi-alpinae, altitud 700-1250 m snm, pedregosidad 5-25\%, pendiente 30-45\%; GFE3: Grupo Fitoecológico 3, asociación Anemono antucensis-Nothofagetum pumilionis, altitud $1250-1350 \mathrm{~m}$ snm, pedregosidad 75-100\%; GFE4: Grupo Fitoecológico 4, asociaciones Carici trichodes-Araucarietum araucanae y Lomatio hirsutae-Nothofagetum antarcticae, altitud > 1350 $\mathrm{m}$ snm, pedregosidad $25-75 \%$, pendiente $45-60 \%$.

Results of the Factorial Correspondence Analysis, using the variables elevation, percentage of stones, slope and plant association. The first two axes represent $24 \%$ of the total variability. GFE1: Phytoecological group 1, association Dasyphyllo diacanthoidis-Nothofagetum alpinae, elevation < 700 masl, stones < $1 \%$, slope < 30\%; GFE2: Phytoecological group 2, associations Nothofagetum procerae and Nothofagetum dombeyi-alpinae, elevation 700-1250 masl, stones 5-25\%, slope 30-45\%; GFE3: Phytoecological group 3, association Anemono antucensis-Nothofagetum pumilionis, elevation 1250-1350 masl, stones 75-100\%; GFE4: Phytoecological group 4, associations Carici trichodes-Araucarietum araucanae and Lomatio hirsutae-Nothofagetum antarcticae, elevation > 1350 masl, stones $25-75 \%$, slope $45-60 \%$.

trato inferior constituido por bambúseas y trepadoras. Se ubica en altitudes medias, en laderas y valles muy húmedos (figura 1, GFE2).

3. Bosque caducifolio micrófilo, con un dosel uniforme de caducifolias, interrumpido por siempreverdes emergentes, que localmente for- 
man bosquetes. Está principalmente ubicado en laderas medias y altas, a menudo con suelos pedregosos y pendientes muy fuertes (figura 1, GFE3).

4. Matorral caducifolio micrófilo, con una fisionomía compleja de matorrales caducifolios altos y achaparrados, con presencia local de árboles siempreverdes, emergentes o en bosquetes. Se desarrolla en mesetas y valles de altitud, también en farellones rocosos y junto a humedales (figura 1, GFE4).

Unidades físicas homogéneas o biotopos. Se determinaron nueve unidades físicas homogéneas o biotopos. Los resultados del AFC entregaron como respuesta que las principales variables que influyen en la distribución de asociaciones son altitud y relieve, expresado este último a través de la pendiente. Desde un punto de vista bioclimático ciertas variables meteorológicas (temperatura, precipitación), expresadas por parámetros e índices, se pueden relacionar estrechamente con los patrones de distribución altitudinal de la vegetación. Según Rivas-Martínez (32), a partir del cálculo del índice de termicidad compensado (Itc) se determinan termotipos, que definen pisos bioclimáticos, expresión integrada por la influencia de la altitud y el relieve sobre el clima local (cuadro 1). A su vez, cada uno de ellos corresponde a un piso vegetacional caracterizado por especies que se desarrollan mejor en las condiciones climáticas dadas $(19,33)$.
Los pisos determinados fueron: colino, montano inferior y montano superior. Los dos últimos corresponden a una subdivisión del piso montano, establecida sobre la base de una marcada diferencia en la vegetación y el Itc. Los límites altitudinales de cada piso son los siguientes: colino, menor a $700 \mathrm{~m} \mathrm{snm}$, montano inferior, entre $700 \mathrm{y}$ $1.300 \mathrm{~m} \mathrm{snm}$, montano superior, entre $1.300 \mathrm{y}$ $1.700 \mathrm{~m} \mathrm{snm}$.

Los pisos bioclimáticos fueron subdivididos en cuatro situaciones de relieve: valles y planos de piedemonte; laderas bajas y laderas medias; altas cumbres y laderas altas, y farellones rocosos.

Se identificaron nueve unidades ambientales homogéneas (cuadro 2, columnas 2, 3 y 4):

1. Colino plano: Se ubica bajo $700 \mathrm{~m}$ snm y corresponde a los valles que bordean el río Malleco. Se caracteriza por suelos profundos, con pendientes suaves a moderadas $(<30 \%)$.

2. Colino de ladera: Se ubica bajo los $700 \mathrm{~m} \mathrm{snm}$, corresponde a laderas bajas y medias, con suelos de profundidad media y pendientes suaves a fuertes (30-60\%).

3. Montano inferior plano: Está entre los 700 y $1.300 \mathrm{~m} \mathrm{snm}$, correspondiente a planos de piedemonte y valles, en torno a los cursos de agua principales. Los sectores correspondientes a los valles se caracterizan por presentar pendientes suaves a moderadas y suelos profundos; son terrenos pantanosos y humedales, denominados localmente "mallines".

\section{CUADRO 1}

Estaciones meteorológicas. T: Temperatura media anual $\left({ }^{\circ} \mathrm{C}\right) ; \mathrm{m}$ : Temperatura media mínima del mes más frío $\left({ }^{\circ} \mathrm{C}\right)$; M: Temperatura media máxima del mes más frío $\left({ }^{\circ} \mathrm{C}\right)$; P: Precipitación media anual (mm) Itc: Indice de termicidad compensado (según Rivas-Martínez et al. (41).

Meteorological stations. T: Annual mean temperature $\left({ }^{\circ} \mathrm{C}\right)$; m: Mean minimum temperature of the coldest month $\left({ }^{\circ} \mathrm{C}\right)$; $\mathrm{M}$ : Mean maximum temperature of the coldest month $\left({ }^{\circ} \mathrm{C}\right)$; P: Mean annual precipitation $(\mathrm{mm})$; Itc: Compensated thermicity index (according to Rivas-Martínez et al. (41).

\begin{tabular}{|c|c|c|c|c|c|c|c|c|}
\hline \multirow[t]{2}{*}{ ESTACION } & \multirow[t]{2}{*}{ Coordenadas } & \multirow{2}{*}{$\begin{array}{l}\text { Altitud } \\
\text { (m snm) }\end{array}$} & \multicolumn{3}{|c|}{ Temperatura $\left({ }^{\circ} \mathrm{C}\right)$} & \multirow{2}{*}{$\begin{array}{c}\mathrm{P} \\
(\mathrm{mm})\end{array}$} & \multirow[t]{2}{*}{ Itc } & \multirow[t]{2}{*}{ Fuente } \\
\hline & & & $\mathrm{T}$ & M & M & & & \\
\hline Ralco & $37^{\circ} 53^{\prime}-71^{\circ} 37^{\prime}$ & 550 & 11,53 & 3,2 & 9,5 & $2.126,8$ & 242,3 & CIREN-CORFO (1990 ) \\
\hline Polcura balseo & $37^{\circ} 19^{\prime}-71^{\circ} 32^{\prime}$ & 740 & 10,26 & 0,4 & 6,8 & $1.087,2$ & 174,6 & $\begin{array}{l}\text { Dirección Meteorológica } \\
\text { de Chile }\end{array}$ \\
\hline Laguna Laja & $37^{\circ} 22^{\prime}-71^{\circ} 22^{\prime}$ & 1.375 & 6,77 & $-0,9$ & 3,0 & $1.023,8$ & 88,7 & $\begin{array}{l}\text { Dirección Meteorológica } \\
\text { de Chile }\end{array}$ \\
\hline
\end{tabular}


4. Montano inferior de ladera: Ubicado entre los 700 y $1.300 \mathrm{~m}$ snm en las laderas bajas y medias, corresponde a la mayor parte de la superficie del área estudiada. Se caracteriza por suelos de profundidad variable, pendientes suaves a fuertes $(30-60 \%)$, considerando laderas tanto de exposición norte como sur.

5. Montano inferior de altas cumbres: Se ubica entre los 700 y $1.300 \mathrm{~m} \mathrm{snm}$, en las altas cumbres y ladera altas. Se caracterizan por ser zonas de pendientes fuertes a muy fuertes (45$100 \%$ ), suelos poco profundos y pedregosos.

6. Montano superior plano: Se encuentra entre los 1.300 y $1.700 \mathrm{~m} \mathrm{snm}$, en valles y planos intermedios. Las pendientes son suaves a moderadas $(0-45 \%)$, con suelos delgados de pedregosidad media.

7. Montano superior de ladera: Se ubica entre los 1.300 y $1.700 \mathrm{~m}$ snm, en las laderas bajas y medias. Los suelos son delgados, pedregosos, con pendientes fuertes (45-60\%).

8. Montano superior de altas cumbres: Ubicado entre los 1.300 y $1.700 \mathrm{~m} \mathrm{snm}$, en los sectores de altas cumbres y laderas altas. Se caracteriza por suelos muy delgados, alta pedregosidad, con afloramientos rocosos.

9. Montano superior de farellones: Corresponde a los sectores de muy fuertes pendientes (>60\%), con escasa vegetación arbórea, exceptuando algunos individuos aislados de araucaria; unidad poco representada en el área.

Estaciones forestales. Se identificaron catorce estaciones forestales resultantes de la relación de los grupos fitosociológicos y fitoecológicos, respecto a las unidades ambientales homogéneas (cuadro 2).

ESTACION 1: Colino plano con bosque caducifolio mesófilo y abundantes elementos laurifolios bajo dosel. Corresponde a relieves planos de valle y piedemonte, de pendientes suaves a moderadas, con suelos profundos, con buena disponibilidad hídrica, en altitudes inferiores a los $700 \mathrm{~m} \mathrm{snm}$ La comunidad vegetal dominante es Dasyphyllo diacanthoidis-Nothofagetum alpinae, con abundancia de Nothofagus obliqua, Persea lingue y Aextoxicon punctatum.

ESTACION 2: Colino plano con bosque caducifolio mesófilo, pobre en elementos laurifolios.
Muestra relieves planos de valle y de piedemonte, con suelos profundos, de pendientes suaves a moderadas, muy favorables para el desarrollo de la vegetación. La comunidad típica de esta situación corresponde a Nothofagetum procerae. La formación boscosa es de estructura coetánea por bosquetes.

ESTACION 3: Colino de ladera con bosques caducifolio mesófilo mixto, pobre en especies laurifolias en el dosel superior. Presenta relieves de laderas bajas y medias, con pendientes fuertes, pero de suelos profundos, con una gruesa capa de hojarasca. La asociación presente es Nothofagetum procerae, con mayor presencia de Laureliopsis philippiana y Nothofagus dombeyi.

ESTACION 4: Colino de ladera con bosques caducifolio mesófilo y abundantes elementos laurifolios bajo dosel. Relieve de laderas con pendientes moderadas a fuertes, de suelos profundos, con gruesa capa de hojarasca. La asociación presente es Dasyphyllo diacanthoidis-Nothofagetum alpinae, con abundancia de Dasyphyllum diacanthoides. Corresponde a bosques de estructura coetánea biestratificada, en su mayoría densos, oscuros y de gran humedad, con alta presencia de epífitas.

ESTACION 5: Montano inferior plano con matorral caducifolio micrófilo. Corresponde a relieves de valle y planos intermedios, de pendientes suaves a moderadas. Son terrenos de condiciones desfavorables, aunque con suelos profundos, pero casi permanentemente anegados y sujetos a la influencia de masas de aire frío ("mallines"). La comunidad florística característica es Lomatio hirsutae-Nothofagetum antarcticae, con dosel poco denso y frecuencia de arbustos y gramíneas en el sotobosque. Ocasionalmente se encuentra presente Araucaria araucana.

ESTACION 6: Montano inferior plano con bosque caducifolio mesófilo con especies laurifolias poco abundantes. Relieve de pendientes suaves a moderadas y suelos medianamente profundos. La asociación presente es Nothofagetum dombeyialpinae, con presencia de Nothofagus obliqua, у $N$. dombeyi como emergente en el dosel superior. Son bosques complejos, de estructura coetánea por bosquetes, a veces con árboles de gran desarrollo. 
BOSQUE 26(2): 47-56, 2005

Ensayo de una tipología de estaciones forestales en el Parque Nacional Tolhuaca, Chile

\section{CUADRO 2}

Estaciones forestales propuestas para el Parque Nacional Tolhuaca.

Forest stations proposed for the Tolhuaca National Park.

\begin{tabular}{|c|c|c|c|c|c|}
\hline $\begin{array}{c}1 \\
\text { Estación }\end{array}$ & $\begin{array}{c}2 \\
\text { Unidad física }\end{array}$ & $\begin{array}{c}3 \\
\text { Altitud } \\
\text { m snm }\end{array}$ & $\begin{array}{c}4 \\
\text { Relieve }\end{array}$ & $\begin{array}{c}5 \\
\text { Formación vegetal }\end{array}$ & $\begin{array}{c}6 \\
\text { Asociaciones vegetales }\end{array}$ \\
\hline 1 & Colino plano & $<700$ & $\begin{array}{l}\text { Valle y planos de } \\
\text { piedemonte }\end{array}$ & $\begin{array}{l}\text { Bosque caducifolio } \\
\text { mesófilo }\end{array}$ & $\begin{array}{l}\text { Dasyphyllo diacanthoidis- } \\
\text { Nothofagetum alpinae }\end{array}$ \\
\hline 2 & Colino plano & $<700$ & $\begin{array}{l}\text { Valle y planos de } \\
\text { piedemonte }\end{array}$ & $\begin{array}{l}\text { Bosque caducifolio } \\
\text { mesófilo }\end{array}$ & Nothofagetum procerae \\
\hline 3 & Colino de ladera & $<700$ & $\begin{array}{l}\text { Laderas bajas } \\
\text { y medias }\end{array}$ & $\begin{array}{l}\text { Bosque caducifolio } \\
\text { mesófilo }\end{array}$ & Nothofagetum procerae \\
\hline 4 & Colino de ladera & $<700$ & $\begin{array}{l}\text { Laderas bajas } \\
\text { y medias }\end{array}$ & $\begin{array}{l}\text { Bosque caducifolio } \\
\text { mesófilo }\end{array}$ & $\begin{array}{l}\text { Dasyphyllo diacanthoidis- } \\
\text { Nothofagetum alpinae }\end{array}$ \\
\hline 5 & $\begin{array}{l}\text { Montano inferior } \\
\text { plano }\end{array}$ & $700-1.300$ & $\begin{array}{l}\text { Valle y planos } \\
\text { de piedemonte }\end{array}$ & $\begin{array}{l}\text { Matorral caducifolio } \\
\text { micrófilo }\end{array}$ & $\begin{array}{l}\text { Lomatio hirsutae-Nothofagetum } \\
\text { antarcticae }\end{array}$ \\
\hline 6 & $\begin{array}{l}\text { Montano inferior } \\
\text { plano }\end{array}$ & $700-1.300$ & $\begin{array}{l}\text { Valle y planos } \\
\text { de piedemonte }\end{array}$ & $\begin{array}{l}\text { Bosque caducifolio } \\
\text { mesófilo }\end{array}$ & Nothofagetum dombeyi-alpinae \\
\hline 7 & $\begin{array}{l}\text { Montano inferior } \\
\text { de ladera }\end{array}$ & $700-1.300$ & $\begin{array}{l}\text { Laderas bajas } \\
\text { y medias }\end{array}$ & $\begin{array}{l}\text { Bosque siempreverde } \\
\text { micrófilo }\end{array}$ & $\begin{array}{l}\text { Lomatio hirsutae-Nothofagetum } \\
\text { antarcticae Nothofagetum } \\
\text { dombeyi-alpinae Carici } \\
\text { trichodes-Araucarietum } \\
\text { araucanae }\end{array}$ \\
\hline 8 & $\begin{array}{l}\text { Montano inferior } \\
\text { de ladera }\end{array}$ & $700-1.300$ & $\begin{array}{l}\text { Laderas bajas } \\
\text { y medias }\end{array}$ & $\begin{array}{l}\text { Bosque caducifolio } \\
\text { mesófilo }\end{array}$ & $\begin{array}{l}\text { Nothofagetum procerae } \\
\text { Nothofagetum dombeyi-alpinae }\end{array}$ \\
\hline 9 & $\begin{array}{l}\text { Montano inferior } \\
\text { de altas cumbres }\end{array}$ & $700-1.300$ & $\begin{array}{l}\text { Altas cumbres } \\
\text { y laderas altas }\end{array}$ & $\begin{array}{l}\text { Matorral caducifolio } \\
\text { micrófilo }\end{array}$ & $\begin{array}{l}\text { Lomatio hirsutae-Nothofagetum } \\
\text { antarcticae Nothofagetum dombeyi- } \\
\text { alpinae Carici trichodes- } \\
\text { Araucarietum araucanae }\end{array}$ \\
\hline 10 & $\begin{array}{l}\text { Montano inferior } \\
\text { de altas cumbres }\end{array}$ & $700-1.300$ & $\begin{array}{l}\text { Altas cumbres } \\
\text { y laderas altas }\end{array}$ & $\begin{array}{l}\text { Bosque caducifolio } \\
\text { mesófilo }\end{array}$ & Nothofagetum dombeyi-alpinae \\
\hline 11 & $\begin{array}{l}\text { Montano superior } \\
\text { plano }\end{array}$ & $1.300-1.700$ & $\begin{array}{l}\text { Valles y planos } \\
\text { de piedemonte }\end{array}$ & $\begin{array}{l}\text { Bosque caducifolio } \\
\text { micrófilo }\end{array}$ & $\begin{array}{l}\text { Anemono antucensis-Nothofagetum } \\
\text { pumilionis }\end{array}$ \\
\hline 12 & $\begin{array}{l}\text { Montano superior } \\
\text { de ladera }\end{array}$ & $1.300-1.700$ & $\begin{array}{l}\text { Altas cumbres } \\
\text { y laderas altas }\end{array}$ & $\begin{array}{l}\text { Bosque caducifolio } \\
\text { micrófilo }\end{array}$ & $\begin{array}{l}\text { Anemono antucensis-Nothofagetum } \\
\text { pumilionis Nothofagetum dombeyi- } \\
\text { alpinae Lomatio hirsutae- } \\
\text { Nothofagetum antarcticae Carici } \\
\text { trichodes-Araucarietum araucanae }\end{array}$ \\
\hline 13 & $\begin{array}{l}\text { Montano superior } \\
\text { de altas cumbres }\end{array}$ & $1.300-1.700$ & $\begin{array}{l}\text { Altas cumbres } \\
\text { y laderas altas }\end{array}$ & $\begin{array}{l}\text { Bosque siempreverde } \\
\text { micrófilo }\end{array}$ & $\begin{array}{l}\text { Lomatio hirsutae-Nothofagetum } \\
\text { antarcticae Carici trichodes } \\
\text { Araucarietum araucanae Anemono } \\
\text { antucensis-Nothofagetum pumiliae }\end{array}$ \\
\hline 14 & $\begin{array}{l}\text { Montano superior } \\
\text { de farellones }\end{array}$ & $1.300-1.700$ & Farellones rocosos & $\begin{array}{l}\text { Matorral caducifolio } \\
\text { micrófilo }\end{array}$ & $\begin{array}{l}\text { Lomatio hirsutae-Nothofagetum } \\
\text { antarcticae Carici trichodes } \\
\text { Araucarietum araucanae }\end{array}$ \\
\hline
\end{tabular}


OSQUE 26(2): 47-56, 2005

Ensayo de una tipología de estaciones forestales en el Parque Nacional Tolhuaca, Chile

ESTACION 7: Montano inferior de ladera con bosques siempreverdes micrófilos, donde la forma de vida fisionómicamente dominante es Nothofagus dombeyi, pero con participación abundante de especies caducifolias mesófilas y matorrales caducifolios micrófilos. Se caracteriza por un relieve de pendientes fuertes y suelos poco profundos, en situaciones locales húmedas y frías. Las asociaciones presentes son Nothofagetum dombeyi-alpinae, Carici trichodes-Araucarietum araucanae, a veces con ocurrencia abundante de N. pumilio, y Lomatio hirsutae-Nothofagetum antarcticae.

ESTACION 8: Montano inferior de ladera con bosque caducifolio mesófilo rico en especies laurifolias. Presenta relieves de pendientes fuertes y suelos delgados. Las comunidades características son Nothofagetum procerae con ocurrencia frecuente de N. dombeyi y N. obliqua, y Nothofagetum dombeyi-alpinae, con abundancia de Laureliopsis philippiana Weinmannia trichosperma y presencia de Myrceugenia chrysocarpa en el sotobosque.

ESTACION 9: Montano inferior de altas cumbres con matorral caducifolio micrófilo y bosque siempreverde. Se caracteriza por laderas de pendientes muy fuertes, suelos delgados con material parental poco intemperizado, con afloramientos rocosos en algunos sectores. Las asociaciones vegetales presentes son Lomatio hirsutae-Nothofagetum antarcticae, Carici trichodes-Araucarietum araucanae, con presencia local abundante de N. pumilio, y Nothofagetum dombeyi-alpinae. Las formaciones boscosas son poco densas y a menudo con individuos de escaso desarrollo.

ESTACION 10: Montano inferior de altas cumbres con bosque caducifolio mesófilo y moderada presencia de especies laurifolias. Relieve de pendientes muy fuertes, con suelos delgados y ocurrencia local de afloramientos rocosos. Las asociaciones presentes son Nothofagetum dombeyi-alpinae, con $N$. dombeyi generalmente abundante en el dosel superior, y presencia importante de Laureliopsis philippiana y Weinmannia trichosperma y un sotobosque denso de Gaultheria phyllireifolia.

ESTACION 11: Montano superior plano con bosque caducifolio micrófilo. Presenta relieve plano a escasa pendiente, con suelos de incipiente desarrollo del perfil, localmente muy delgados. La co- munidad característica es Anemono antucensisNothofagetum pumilionis, con presencia ocasional de Araucaria araucana. El sotobosque es dominado por un dosel denso de regeneración de $N$. pumilio.

ESTACION 12: Montano superior de ladera con bosques y matorrales caducifolios micrófilos, con especies siempreverdes. Presenta relieves de pendientes moderadas, con suelos delgados pedregosos, con manifestaciones de erosión geológica. Las asociaciones presentes son Anemono antucensisNothofagetum pumilionis, Carici trichodes- Araucarietum araucanae, Nothofagetum dombeyi-alpinae y Lomatio hirsutae-Nothofagetum antarcticae. En el sotobosque son frecuentes las especies arbustivas laurifolias, como Myrceugenia chrysocarpa y Drimys andina.

ESTACION 13: Montano superior de altas cumbres con bosque siempreverde micrófilo con dominancia fisionómica de Araucaria araucana. Presenta relieves de pendientes muy fuertes, en laderas altas y cumbres abruptas, con suelos de escaso desarrollo y abundante pedregosidad. Las comunidades representativas son Anemono antucensisNothofagetum pumilionis, Carici trichodesAraucarietum araucanae y Lomatio hirsutae-Nothofagetum antarcticae. Localmente hay presencia de Nothofagus dombeyi y N. obliqua; es frecuente que tanto $N$. pumilio como $N$. antarctica crezcan con hábito arbustivo e intrincado.

ESTACION 14: Montano superior de farellones con matorral caducifolio micrófilo y bosquetes muy ralos de Araucaria araucana. Corresponde a un relieve de farellones rocosos y cumbres escarpadas, con fuertes pendientes y substratos rocosos. Las comunidades presentes corresponden a $\mathrm{Lo}$ matio hirsutae-Nothofagetum antarcticae y Carici trichodes-Araucarietum araucanae.

\section{CONCLUSIONES}

Sobre la base de la aplicación de índices es posible establecer pisos bioclimáticos. La relación de estos pisos con la posición topográfica en el relieve montañoso propio del área estudiada permiten fundamentar la definición de ambientes físicos homogéneos o biotopos. Estos tipos de am- 
BOSQUE 26(2): 47-56, 2005 Ensayo de una tipología de estaciones forestales en el Parque Nacional Tolhuaca, Chile

bientes físicos demuestran tener incidencia en la distribución de la vegetación en el nivel de las cuatro formaciones vegetales que se definen para el área.

El análisis de la distribución local de las comunidades vegetales, definidas sobre una base fitosociológica, resulta de difícil interpretación. Sería dable esperar que existiera una relación unívoca biotopo-asociación, tal como lo establece la teoría fitosociológica. Pero es posible encontrar en el área en estudio a dos o más asociaciones bajo la influencia de las mismas condiciones ambientales. La explicación posible es que el filtro de definición de los biotopos no es lo suficientemente fino como para poder discriminar con mayor seguridad entre estados de factores ecológicos muy similares.

El análisis de la distribución de comunidades y formaciones vegetacionales respecto a los gradientes ambientales de la denominada "pluvisilva valdiviana" en el sentido de Hauman (34), Schmithüsen (35) y Hueck (36), deja aún muchos interrogantes y no permite fijar marcos de referencia sistemáticos en términos de interpretación fitosociológica $(14,27,37,38)$. No existen reconocimientos de suelos suficientemente detallados y la disponibilidad de estaciones meteorológicas es baja, en especial en sectores de altitud. Al mismo tiempo, no hay aún estudios suficientes sobre la naturaleza genética de las especies dominantes, con probable existencia de subespecies, variedades y ecotipos aún no bien identificados $(39,40)$.

Aunque con cierto grado de dificultad, resulta posible establecer unidades homogéneas o estaciones forestales tal como las define Brêthes (12), identificables en terreno y que podrían sentar las bases para una sistematización de los tratamientos silviculturales y de las medidas necesarias para una correcta gestión forestal (17).

\section{AGRADECIMIENTOS}

Se agradece a la Corporación Nacional Forestal (CONAF-Chile) y a la Office National des Forêts (ONF-Francia), por el apoyo brindado en terreno; a los revisores anónimos por sus sugerencias, y a los doctores W. Pollmann y U. Eskuche, por el envío de literatura relevante.

\section{BIBLIOGRAFIA}

(1) WHITTAKER, R.H. (ed). Ordination and classification of communities. The Hague: Dr. W. Junk Publishers 1973. 737 p.

(2) KUCHLER, A.W., I.S. ZONNEVELD (eds.). Vegetation mapping. Dordrecht: Kluwer Academic Publishers. 1988. $635 \mathrm{p}$

(3) KENT, M., P. COKER. Vegetation description and analysis. A practical approach. Chichester: John Wiley and Sons. 1995. 463 p.

(4) FREY, T.E.A. The finish school and forest site-types In: WHITTAKER, R.H. (ed). Ordination and classification of communities. The Hague: Dr. W. Junk Publishers. 1973. p. 403-433.

5) PFISTER, R.D., S.F. ARNO. Classification of forest habitats based on potential climax vegetation. Forest Science (U.S.A.), 1980, vol. 26, p. 52-70.

(6) BELAND, M., Y. BERGERON, B. HARVEY, D. ROBERT. Québec's ecological framework for forest management: a case study in the boreal forest of Abitibi. Forest Ecology and Management (Holanda), 1992, vol. 49, p. 247-266.

(7) PHILIP, M.S. Measuring trees and forests. Cambridge: Cabi Publishing, University Press. 1998. 310 p.

(8) HILLS, G.A. Comparison of forest ecosystem (vegetation and soil) in different climatic zones. Silva Fennica (Finlandia), 1960, vol. 105, p. 33-39.

(9) CAJANDER, A.K. The theory of forest types. Acta Forestalia Fennica (Finlandia), 1926, vol. 29, No 3 , p. 1-107.

(10) DUCHAUFOUR, PH., M. BONNEAU, E.-F. DEBAZAC, J. PARDE. Types de forêt et aménagement: la Forêt de la Controlerie en Argonne. Annales de l'École Nationale des Eaux et Forêts et de la Station de Recherches et Expériences (Francia), 1961, vol. 18, No 1, p. 1-44.

(11) BONNEAU, M., J. TIMBAL. Définition et cartographie des stations. Conceptions françaises et étrangères. An nales des Sciences Forestières (Francia), 1973, vol. 30, $\mathrm{N}^{\circ} 3$, p. 201-218.

(12) BRÊTHES, A. La typologie des stations forestières. Recommandations méthodologiques. Revue Forestière Française (Francia), 1989, vol. 41, No 1, p. 7-27.

(13) DONOSO, C. Tipos forestales de los bosques nativos de Chile. Santiago: Conaf/Pnud/Fao, Programa de Investigación y Desarrollo Forestal, Documento de Trabajo $\mathrm{N}^{\mathrm{o}}$ 38. 1981. $82 \mathrm{p}$.

(14) DONOSO, C. Bosques templados de Chile y Argentina. Variación, estructura y dinámica. $1^{\text {a }}$ edición. Santiago: Editorial Universitaria. 1993. 484 p.

(15) VITA, A. Los tratamientos silviculturales. Santiago: Facultad de Ciencias Agrarias y Forestales, Universidad de Chile. 1996. 147 p.

(16) PERTHUISOT, N. Réalisation d'une première typologie forestière dans la Reserve de Malleco (Chili, région centre-sud) et proposition de lignes d'aménagement. Temuco: Conaf/Onf/Engref. Rapport de fin d'études, marsaoût 1995. 1995. 58 p.

(17) ESTAY, M. Determinación de una tipología de estaciones forestales en el Parque Nacional Tolhuaca, IX Región. Santiago: Memoria Ing. Forestal, Universidad de Chile, Facultad de Ciencias Forestales. 2000. 76 p.

(18) RAMIREZ, C. Estudio florístico y vegetacional del Parque Nacional Tolhuaca. Santiago: Museo Nacional de Historia Natural (Chile), Publicación Ocasional No 24 1978. p. 3-23. 
Q6(2): 47-56, 2005

Ensayo de una tipología de estaciones forestales en el Parque Nacional Tolhuaca, Chile

(19) AMIGO, J., C. RAMIREZ. A bioclimatic classification of Chile: woodland communities in the temperate zone. Plant Ecology (Holanda), 1998, vol. 136, № 1, p. 9-26.

(20) PERALTA, M. Uso, clasificación y conservación de suelos. Santiago: Ministerio de Agricultura, Servicio Agrícola y Ganadero. 1976. 337 p.

(21) BRAUN-BLAQUET, J. Sociología vegetal. Estudio de las comunidades vegetales. Buenos Aires: Acmé Agency. $1950.444 \mathrm{p}$

(22) MUELLER-DOMBOIS, D., H. ELLENBERG. Aims and methods of vegetation ecology. New York: John Wiley and Sons. 1974. 547 p

(23) LUEBERT, F., R. GAJARDO, M. ESTAY. Nota fitosociológica sobre las comunidades forestales del Parque Nacional Tolhuaca (Chile). Boletín Museo Nacional de Historia Natural (Chile), 2003, vol. 52, p. 51-66.

(24) GAJARDO, R. La vegetación natural de Chile. Clasificación y distribución geográfica. Santiago: Editorial Universitaria. 1994. $165 \mathrm{p}$.

(25) CARO, C. Esquema de caracterización tipológica para los matorrales y bosques esclerófilos chilenos. Santiago: Memoria Ing. Forestal, Universidad de Chile. Facultad de Ciencias Agrarias y Forestales. 1996. 111 p.

(26) OBERDORFER, E. Pflanzensoziologische Studien in Chile. Ein Vergleich mit Europa. Weinheim: Verlag von J. Cramer. 1960. 208 p.

(27) POLLMANN, W. Caracterización florística y posición sintaxonómica de los bosques caducifolios de Nothofagus alpina (Poepp. et Endl.) Oerst. en el centro-sur de Chile. Phytocoenologia (Alemania), 2001, vol. 31, N 3, p. 353-400.

(28) HILDEBRAND-VOGEL, R. Structure and dynamics of Southern Chilean natural forests with special reference to the relationship of evergreen versus deciduous elements. Folia Geobotanica (República Checa), 2002, vol. 37, p. 107-128.

(29) ESKUCHE, U. Fisionomía y sociología de los bosques de Nothofagus dombeyi en la región de Nahuel Huapi. Vegetatio (Holanda), 1968, vol. 16, p. 192-204.

(30) ESKUCHE, U. Berberitzengebüche und Nothofagus antarctica-Wälder in Nordwestpatagonien. Vegetatio (Holanda), 1969, vol. 19, p. 264-285.
(31) HILDEBRAND-VOGEL, R., R. GODOY, A. VOGEL. Subantarctic-Andean Nothofagus pumilio forests. Vegetatio (Holanda), 1990, vol. 89, p. 55-68.

(32) RIVAS - MARTINEZ, S. Clasificación bioclimática de la Tierra. Folia Botánica Matritensis (España), 1993, vol. 10, p. 1-23.

(33) DOUGUEDROIT, A. Comparaison entre modéles d'etagement de la vegetation et de gradient thermique topoclimatique à partir du cas des Alpes du sud. Bulletin de la Société Botanique de France (Francia), 1984 vol. 131, Actualités Botaniques, $N^{\text {os }}$ 2/3/4, p. 181-190.

(34) HAUMAN, L. La forêt valdivienne et ses limites. Recueil de l'Institut Botanique Leo Herrera (Argentina), 1913, vol. 9, p. 346-408.

(35) SCHMITHUSEN, J. Die Räumliche Ordnung der Chilenischen Vegetation. Bonner Geographische Abhandlungen (Alemania), 1956, vol. 17, p. 1-86.

(36) HUECK, K. Los bosques de Sudamérica. Ecología, composición e importancia económica. Eschborn: Ediciones de la Sociedad Alemana de Cooperación Técnica. 1978. $476 \mathrm{p}$.

(37) WEINBERGER, P. Definición de grupos ecológicos en formaciones boscosas siempreverdes de la zona austral de Chile. Bosque (Chile), 1997, vol. 18, No 2, p. 29-41.

(38) WEINBERGER, P., C. RAMIREZ. Microclima y regeneración natural de raulí, roble y coiguie (Nothofagus alpina, Nothofagus obliqua y Nothofagus dombeyi). Bosque (Chile), 2001, vol. 22, $\mathrm{N}^{\circ}$ 1, p. 11-26.

(39) RAMIREZ, C., M.A. CORREA, H. FIGUEROA, J. SAN MARTIN. Variación del hábito y hábitat de Nothofagus antarctica en el sur de Chile. Bosque (Chile), 1985, vol. 6, $\mathrm{N}^{\circ} 2$, p. $55-73$.

(40) VAZQUEZ, F.M., R.A. RODRIGUEZ. A new subspecies and two new combinations of Nothofagus Blume (Nothofagaceae) from Chile. Botanical Journal of the Linnean Society (Inglaterra), 1999, vol. 129, p. 75-83.

(41) RIVAS - MARTINEZ, S., D. SANCHEZ MATTA, M COSTA. North American boreal and western temperate forest vegetation. Itinera Geobotánica (España), 1999, vol. $12, \mathrm{~N}^{\mathrm{o}} 5-316$.

(42) CIREN-CORFO. Atlas agroclimático de Chile: Regiones IV a IX. Santiago: Centro de Información de Recursos Naturales, CORFO. 1990. $67 \mathrm{p}$ 